\title{
Influence of beetroot juice supplementation on intermittent exercise performance
}

\author{
Lee J. Wylie ${ }^{1} \cdot$ Stephen J. Bailey $^{1} \cdot$ James Kelly $^{1} \cdot$ James R. Blackwell $^{1} \cdot$ \\ Anni Vanhatalo ${ }^{1} \cdot$ Andrew M. Jones ${ }^{1}$
}

Received: 20 August 2015 / Accepted: 7 November 2015 / Published online: 27 November 2015

(C) The Author(s) 2015. This article is published with open access at Springerlink.com

\begin{abstract}
Purpose This study tested the hypothesis that nitrate $\left(\mathrm{NO}_{3}{ }^{-}\right)$supplementation would improve performance during high-intensity intermittent exercise featuring different work and recovery intervals.

Method Ten male team-sport players completed highintensity intermittent cycling tests during separate 5-day supplementation periods with $\mathrm{NO}_{3}{ }^{-}$-rich beetroot juice (BR; $8.2 \mathrm{mmol} \mathrm{NO}_{3}{ }^{-} \mathrm{day}^{-1}$ ) and $\mathrm{NO}_{3}{ }^{-}$-depleted beetroot juice (PL; $0.08 \mathrm{mmol} \mathrm{NO}_{3}{ }^{-} \mathrm{day}^{-1}$ ). Subjects completed: twentyfour 6-s all-out sprints interspersed with $24 \mathrm{~s}$ of recovery $(24 \times 6$-s); seven 30 -s all-out sprints interspersed with $240 \mathrm{~s}$ of recovery $(7 \times 30$-s); and six 60-s self-paced maximal efforts interspersed with $60 \mathrm{~s}$ of recovery $(6 \times 60$-s); on days 3,4 , and 5 of supplementation, respectively.

Result Plasma $\left[\mathrm{NO}_{2}^{-}\right.$] was $237 \%$ greater in the BR trials. Mean power output was significantly greater with BR relative to $\mathrm{PL}$ in the $24 \times 6$-s protocol $(568 \pm 136$ vs. $539 \pm 136 \mathrm{~W} ; P<0.05)$, but not during the $7 \times 30$-s $(558 \pm 95$ vs. $562 \pm 94 \mathrm{~W})$ or $6 \times 60$-s $(374 \pm 57$ vs. $375 \pm 59 \mathrm{~W})$ protocols $(P>0.05)$. The increase in blood [lactate] across the $24 \times 6$-s and $7 \times 30$-s protocols was greater with BR $(P<0.05)$, but was not different in the $6 \times 60$-s protocol $(P>0.05)$.

Conclusion BR might be ergogenic during repeated bouts of short-duration maximal-intensity exercise interspersed with short recovery periods, but not necessarily during
\end{abstract}

Communicated by David C. Poole.

Andrew M. Jones

a.m.jones@exeter.ac.uk

Sport and Health Sciences, College of Life and Environmental Sciences, St. Luke's Campus, University of Exeter, Heavitree Road, Exeter, Devon EX1 2LU, UK longer duration intervals or when a longer recovery duration is applied. These findings suggest that BR might have implications for performance enhancement during some types of intermittent exercise.

Keywords Nitric oxide $\cdot$ Beetroot juice $\cdot$ Repeated sprint exercise $\cdot$ Exercise performance $\cdot$ Team sports

\begin{tabular}{|c|c|}
\hline \multicolumn{2}{|c|}{ Abbreviations } \\
\hline ANOVA & Analysis of variance \\
\hline ATP & Adenosine triphosphate \\
\hline BR & $\mathrm{NO}_{2}^{-}$-rich beetroot juice \\
\hline LSD & Least significant difference \\
\hline MPO & Mean power output \\
\hline $\mathrm{MPO}_{\text {mean }}$ & Mean of mean power output \\
\hline NO & Nitric oxide \\
\hline $\mathrm{NO}_{2}^{-}$ & Nitrite \\
\hline $\mathrm{NO}_{3}^{-}$ & Nitrate \\
\hline NOS & Nitric oxide synthase \\
\hline $\mathrm{O}_{2}$ & Oxygen \\
\hline $\mathrm{PCr}$ & Phosphocreatine \\
\hline PL & $\mathrm{NO}_{3}^{-}$-depleted beetroot juice \\
\hline $\mathrm{PO}_{2}$ & Partial pressure of oxygen \\
\hline PPO & Peak power output \\
\hline $\mathrm{PPO}_{\text {mean }}$ & Mean of peak power output \\
\hline Rpm & Revolutions per minute \\
\hline$\dot{V} \mathrm{O}_{2}$ & Oxygen uptake \\
\hline$\dot{V} \mathrm{O}_{2 \text { peak }}$ & Peak oxygen uptake \\
\hline$\Delta$ & Change \\
\hline
\end{tabular}

\section{Introduction}

Nitric oxide (NO) is a multi-functional physiological signaling molecule that can be endogenously derived from 
the oxygen $\left(\mathrm{O}_{2}\right)$-dependent catabolism of L-arginine in a reaction catalyzed by the NO synthase (NOS) enzymes (Stamler and Meissner 2001), or from the $\mathrm{O}_{2}$-independent reduction of nitrite $\left(\mathrm{NO}_{2}{ }^{-}\right)$by numerous $\mathrm{NO}_{2}{ }^{-}$reductases (Lundberg and Weitzberg 2009). In recreationally active and moderately trained subjects, increasing the circulating plasma $\left[\mathrm{NO}_{2}{ }^{-}\right]$via inorganic nitrate $\left(\mathrm{NO}_{3}{ }^{-}\right)$supplementation has been reported to improve performance during sub-maximal endurance exercise in most (e.g., Bailey et al. 2009; Cermak et al. 2012; Porcelli et al. 2015; Wylie et al. 2013a; see Jones 2014 for review), but not all (e.g., Kelly et al. 2014), previous studies. However, the reduction of $\mathrm{NO}_{2}{ }^{-}$to $\mathrm{NO}$, and therefore the potential for NO-mediated physiological signaling following $\mathrm{NO}_{3}{ }^{-}$supplementation, is potentiated as $\mathrm{O}_{2}$ tension (Castello et al. 2006) and $\mathrm{pH}$ (Modin et al. 2001) decline. Given that muscle $\mathrm{PO}_{2}$ and $\mathrm{pH}$ decline to a greater extent (Richardson et al. 1995) at higher exercise intensities, $\mathrm{NO}_{3}{ }^{-}$supplementation may be more likely to increase NO synthesis, and perhaps improve performance, at higher exercise intensities.

Fatigue development during high-intensity intermittent exercise is linked, in part, to the decline in muscle phosphocreatine concentration [PCr] (Fulford et al. 2013; Gaitanos et al. 1993), whereas recovery of intermittent exercise performance is linked to muscle $\mathrm{PCr}$ resynthesis (Bogdanis et al. 1995, 1996; Mendez-Villanueva et al. 2012). Importantly, $\mathrm{NO}_{3}{ }^{-}$supplementation has been shown to lower the $\mathrm{PCr}$ cost of force production during high-intensity intermittent exercise (Fulford et al. 2013), which might delay the attainment of a critically low muscle [PCr] during intermittent exercise (Chidnok et al. 2013). Moreover, the increased perfusion and oxygenation of type II muscle that has been reported following $\mathrm{NO}_{3}{ }^{-}$supplementation (Ferguson et al. 2013, 2015) might facilitate the $\mathrm{O}_{2}$-dependent recovery of PCr (Trump et al. 1996; Vanhatalo et al. 2011) in the type II muscle fibers that are preferentially recruited during highintensity intermittent exercise (Essén 1978; Green 1978; Krustrup et al. 2004, 2009; Thomson et al. 1979). Supplementation with $\mathrm{NO}_{3}{ }^{-}$has also been reported to: enhance calcium handling and augment the rate of force development in type II muscle fibers (Hernández et al. 2012); and to increase force production (Coggan et al. 2015; Haider and Folland 2014) and attenuate fatigue development at high muscle contraction frequencies (Bailey et al. 2015) and when the proportional contribution of type II muscle to force production is expected to be increased (Breese et al. 2013). These findings suggest that $\mathrm{NO}_{3}{ }^{-}$supplementation might improve performance during high-intensity intermittent exercise. Moreover, given that the recovery of muscle $\mathrm{PCr}$ and force production during intermittent exercise is incomplete when shorter recovery durations are applied (Bogdanis et al. 1995), it is possible that $\mathrm{NO}_{3}{ }^{-}$supplementation might be most effective during high-intensity intermittent exercise when the recovery duration between work intervals is relatively short.

Given that $\mathrm{NO}_{3}{ }^{-}$supplementation has been shown to improve (Aucouturier et al. 2015; Bond et al. 2012; Thompson et al. 2015; Wylie et al. 2013a), compromise (Martin et al. 2014) or have no effect (Christensen et al. 2013; Muggeridge et al. 2013) on high-intensity intermittent exercise performance, the potential for this supplement to be ergogenic in intermittent exercise is controversial. These equivocal findings might be attributed to inter-study differences in the training status of the participants, the exercise modality employed, the $\mathrm{NO}_{3}{ }^{-}$supplementation procedures, and differences in the intermittent exercise protocols (work and rest intensities, work and rest durations, work-to-rest ratio, and number of work intervals). Further research is required to elucidate the relative efficacy of $\mathrm{NO}_{3}{ }^{-}$supplementation in enhancing performance in different intermittent exercise performance protocols.

The purpose of this investigation was to assess the effects of $\mathrm{NO}_{3}{ }^{-}$supplementation on performance during a variety of high-intensity intermittent exercise tests using the same subject population, exercise modality, and $\mathrm{NO}_{3}{ }^{-}$dosing procedures. We hypothesized that: (1) $\mathrm{NO}_{3}{ }^{-}$ supplementation would improve performance during all high-intensity intermittent protocols administered and (2) performance would be enhanced to the greatest extent when maximal-intensity intermittent exercise was accompanied by the shortest recovery duration.

\section{Methods}

\section{Subjects}

Ten male recreational team-sport players (mean \pm SD: age $21 \pm 1$ years, body mass $87.5 \pm 9.5 \mathrm{~kg}$, height $1.82 \pm 0.01 \mathrm{~m}, \dot{V} \mathrm{O}_{2 \text { peak }}, 58 \pm 8 \mathrm{~mL} \mathrm{~kg}^{-1} \mathrm{~min}^{-1}$ ) familiar with intense intermittent exercise volunteered to participate in this study. Prior to testing, subjects were informed of the protocol and the possible risks and benefits of participation before written informed consent was obtained. All procedures were approved by the Institutional Ethics Committee and conformed to the code of ethics of the Declaration of Helsinki.

\section{Experimental design}

The subjects reported to the laboratory on eight separate occasions over a $\sim 4$-week period. During the first visit to the laboratory, subjects initially performed a ramp incremental test for assessment of ramp test peak power and peak $\dot{V} \mathrm{O}_{2}\left(\dot{V} \mathrm{O}_{2 \text { peak }}\right)$. After $30 \mathrm{~min}$ of passive recovery, subjects completed $5 \times 6$-s all-out cycle sprints interspersed 
by $24 \mathrm{~s}$ of recovery and, following an additional $10 \mathrm{~min}$ of passive recovery, $2 \times 30$-s all-out cycle efforts interspersed by $4 \mathrm{~min}$ of recovery. After a minimum of $24 \mathrm{~h}$ recovery, subjects returned to the laboratory to complete $6 \times 60$-s self-paced maximal efforts separated by $60 \mathrm{~s}$ of active recovery. These sprints and self-paced efforts served as a familiarization to the three experimental protocols that are outlined in detail below.

Following completion of the preliminary testing, subjects were assigned in a randomised, double-blind, crossover experimental design to receive either $\mathrm{NO}_{3}{ }^{-}$-rich beetroot juice (BR) or $\mathrm{NO}_{3}{ }^{-}$-depleted beetroot juice (PL) for 5 days. Subjects completed $24 \times 6$-s all-out sprints, $7 \times 30$-s all-out sprints, and $6 \times 60$-s self-paced maximal efforts on days 3, 4, and 5 of supplementation, respectively. A washout period of at least 7 days separated each supplementation period. Subjects were asked to record their food intake in the $24 \mathrm{~h}$ preceding the first experimental trial and to replicate this same diet in the $24 \mathrm{~h}$ preceding all subsequent trials. Subjects were instructed to arrive at the laboratory in a rested and fully hydrated state, at least $3 \mathrm{~h}$ post-prandial, and to avoid strenuous exercise in the $24 \mathrm{~h}$ preceding each testing session. Subjects were also asked to refrain from caffeine and alcohol intake 6 and $24 \mathrm{~h}$ before each laboratory visit, respectively, and to abstain from antibacterial mouthwash and chewing gum use throughout the study as these products blunt the reduction of nitrate to nitrite in the oral cavity (Govoni et al. 2008). All tests were performed at the same time of day $( \pm 1 \mathrm{~h})$.

\section{Determination of peak oxygen uptake and ramp test peak power}

All exercise tests were performed on an electrically braked cycle ergometer (Lode Excalibur Sport, Groningen, The Netherlands). The ergometer saddle and handle bar height configuration was recorded and reproduced in all subsequent tests. The ramp incremental test protocol consisted of 3 min of "unloaded" baseline cycling at $20 \mathrm{~W}$ followed by a linear $30 \mathrm{~W} \mathrm{~min}{ }^{-1}$ increase in power output until volitional exhaustion. Subjects were instructed to maintain a cadence of $80 \mathrm{rpm}$ and, when cadence fell below $70 \mathrm{rpm}$ despite strong verbal encouragement, the test was terminated and peak power was recorded. Pulmonary gas exchange was measured throughout the test on a breath-bybreath basis and subsequently averaged into 10-s bins for analysis. $\dot{V} \mathrm{O}_{2 \text { peak }}$ was calculated as the mean value over the final $30 \mathrm{~s}$ of exercise.

\section{Intermittent exercise tests}

Before the onset of all intermittent exercise tests, subjects completed a standardized warm-up that comprised 5 min of cycling at $80 \mathrm{~W}(80 \mathrm{rpm})$, followed immediately by $3 \times 3$-s sprints, each interspersed by $20 \mathrm{~s}$ of passive recovery. Five minutes after completing the warm-up protocol, subjects performed one of the individual intermittent protocols as described below.

\section{$24 \times 6$-s protocol}

After undergoing the standardized warm-up, subjects completed a single 6-s all-out sprint. The mean power output (MPO) recorded in this 6-s sprint was used as a criterion score during the subsequent $24 \times 6$-s cycle test. Upon completion of this sprint, subjects rested for $5 \mathrm{~min}$ before completing the $24 \times 6$-s test, which consisted of 24 6-s all-out sprints departing every $30 \mathrm{~s}$. To mitigate the potential confounding influence of different pacing strategies between supplements (Waldron and Highton 2014), the MPO during sprint 1 of the $24 \times 6$-s protocol was required to equal or exceed $95 \%$ of the MPO during the benchmark sprint (Fitzsimons et al. 1993). Participants met this criterion in all tests. All 6-s sprints were performed in a standing position and interspersed by $24 \mathrm{~s}$ of passive seated recovery.

\section{$7 \times 30$-s protocol}

The $7 \times 30$-s protocol consisted of seven 30 -s all-out cycle sprints interspersed with 4 min of recovery. Each 4 min recovery period consisted of $210 \mathrm{~s}$ of active recovery at $20 \mathrm{~W}$ followed by $30 \mathrm{~s}$ of passive recovery. Subjects remained seated during all 30-s sprints and recovery periods. During all 6- and 30-s sprints, subjects were verbally encouraged to perform with maximum effort, but were not informed of the sprint number to prevent pacing.

\section{$6 \times 60$-s self-paced protocol}

The $6 \times 60$-s protocol consisted of six 60-s self-paced cycle efforts interspersed with $60 \mathrm{~s}$ of recovery. Subjects were instructed to achieve the highest MPO across all 60-s efforts. Each 60-s recovery period consisted of $40 \mathrm{~s}$ of active recovery at $20 \mathrm{~W}$ ('unloaded') and $20 \mathrm{~s}$ of seated passive recovery. Subjects remained seated during all 60-s self-paced work intervals and recovery periods. Subjects were not provided with any verbal encouragement or information pertaining to the interval number until the final 60-s effort. At this point, subjects were instructed to maximize MPO.

Five seconds before the onset of each work interval, subjects were asked to position the right crank $45^{\circ}$ down from the vertical axis. Subsequently, all work intervals were preceded by a 3-s countdown followed by a clear "GO" command. The resistance during each sprint and self-paced exercise bout was applied using the cadence-dependent 
linear function (linear factor $=$ power/cadence squared) of the Lode ergometer. The fixed resistance for the 6- and 30 -s sprints was set so that upon attaining a cadence of $120 \mathrm{rpm}$, subjects would achieve a power output equivalent to 270 and $220 \%$ of their ramp test peak power, respectively. These resistances were selected, based on findings from pilot experiments, as they allowed subjects to achieve peak power output (PPO) at $\sim 120 \mathrm{rpm}$, which is the optimal cadence for attainment of PPO during all-out cycling exercise (McCartney et al. 1985). The fixed resistance during the 60-s self-paced exercise bouts was set so that subjects would achieve $80 \%$ of their ramp peak power at $80 \mathrm{rpm}$. Subjects were blinded to the elapsed exercise time in the $24 \times 6$-s and $7 \times 30$-s intermittent exercise performance tests.

\section{Supplementation}

Following completion of the pre-supplementation tests, subjects were assigned using a randomised, balanced, cross-over design to receive concentrated $\mathrm{NO}_{3}{ }^{-}$-rich beetroot juice (BR; containing $\sim 4.1 \mathrm{mmol}$ of $\mathrm{NO}_{3}{ }^{-}$per $70 \mathrm{~mL}$; Beet It, James White Drinks Ltd., Ipswich, UK) and $\mathrm{NO}_{3}{ }^{-}$depleted beetroot juice (PL; containing $\sim 0.04 \mathrm{mmol} \mathrm{NO}_{3}{ }^{-}$ per $70 \mathrm{~mL}$; Beet It, James White Drinks Ltd., Ipswich, UK) for 5 days. On non-experimental days (days 1 and 2) of each supplementation period, subjects consumed $1 \times 70 \mathrm{~mL}$ in the morning ( 10 a.m.) and $1 \times 70 \mathrm{~mL}$ in the evening ( $\sim 7$ p.m.). On experimental days (days 3,4 , and 5 of supplementation), subjects consumed $2 \times 70 \mathrm{~mL}$ $2.5 \mathrm{~h}$ prior to the onset of testing procedures. This dose of $\mathrm{BR}$ and the timing of ingestion was selected based on our previous research which suggested that $8.4 \mathrm{mmol}$ of $\mathrm{NO}_{3}{ }^{-}$ (administered as $140 \mathrm{~mL} \mathrm{BR}$ ) resulted in a peak increase in plasma [nitrite] 2-3 h later that coincided with improved exercise tolerance (Wylie et al. 2013a). On experimental days 1 and 2 for both BR and PL (days 3 and 4 of supplementation), subjects consumed a further $1 \times 70 \mathrm{~mL}$ dose $3 \mathrm{~h}$ post-completion of testing procedures.

\section{Measurements and data analysis procedures}

\section{Performance variables}

Power output was recorded continuously at $1 \mathrm{~Hz}$ throughout each exercise test using customized software (created through Labview) and exported for subsequent analysis. For the $24 \times 6$-s and $7 \times 30$-s tests, MPO and PPO were calculated for each sprint. In addition, the mean of MPO $\left(\mathrm{MPO}_{\text {mean }}\right)$ was calculated for each protocol and mean of PPO $\left(\mathrm{PPO}_{\text {mean }}\right)$ was calculated for the $24 \times 6$-s and $7 \times 30$-s protocols. To assess performance at different stages of the $24 \times 6$-s protocol, MPO and PPO data were pooled into bins of six sprints (i.e. sprints $1-6,7-12$, 13-18, 19-24) prior to statistical analysis.

\section{Pulmonary gas exchange and ventilation}

Pulmonary gas exchange and ventilation were collected breath-by-breath in all exercise tests. Subjects wore a nose clip and breathed through a low-dead space $(90 \mathrm{~mL})$, low-resistance $\left(0.75 \mathrm{mmHg} \mathrm{L}^{-1} \mathrm{~s}^{-1}\right.$ at $\left.15 \mathrm{~L} / \mathrm{s}^{-1}\right)$ mouthpiece and impeller turbine assembly (Jaeger Triple V). The inspired and expired gas concentration signals were continuously sampled using paramagnetic $\left(\mathrm{O}_{2}\right)$ and infrared $\left(\mathrm{CO}_{2}\right)$ analyzers (Oxycon Pro; Jaeger, Hoechberg, Germany) via a capillary line connected to the mouthpiece. These analyzers were calibrated before each test with gases of known concentration, and the turbine volume transducer was calibrated using a 3-L syringe (Hans Rudolph, Kansas City, MO). Breath-by-breath $\dot{V} \mathrm{O}_{2}$ data from each test were linearly interpolated to provide second-by-second values. Subsequently, mean $\dot{V} \mathrm{O}_{2}$ was assessed during each work and recovery period and averaged to provide the overall mean $\dot{V} \mathrm{O}_{2}$ during the work and recovery periods for each intermittent exercise test. The mean $\dot{V} \mathrm{O}_{2}$ across all interval and recovery periods for each intermittent exercise test was also calculated.

\section{Venous and capillary blood sampling}

Upon arrival at the laboratory for each intermittent exercise test, a venous blood sample was taken for the determination of plasma $\left[\mathrm{NO}_{2}{ }^{-}\right]$. Venous blood samples were drawn into $7.5 \mathrm{~mL}$ lithium-heparin tubes (Monovette lithium heparin; Sarstedt, Leicester, UK). Within 1 min of collection, samples were centrifuged at $4000 \mathrm{rpm}$ and $4{ }^{\circ} \mathrm{C}$ for $7 \mathrm{~min}$. Plasma was subsequently aliquoted and immediately frozen at $-80{ }^{\circ} \mathrm{C}$ for later analysis of $\left[\mathrm{NO}_{2}{ }^{-}\right]$as described previously (Wylie et al. 2013a, b).

Capillary blood samples were collected from a fingertip into a capillary tube prior to the warm-up procedure and $20 \mathrm{~s}$ prior to the onset of each exercise test. Additionally, capillary blood samples were collected after every two sprints in the $24 \times 6$-s protocol and after every exercise interval in the $7 \times 30$-s and $6 \times 60$-s protocols. These samples were stored on ice and analyzed within $5 \mathrm{~min}$ of collection to determine blood lactate concentration [lactate] using an automated blood [lactate] analyzer (YSI 1500; Yellow Springs Instrument, Yellow Springs, $\mathrm{OH}$ ).

\section{Statistical analysis}

Between-supplement differences in $\mathrm{MPO}_{\text {mean }}, \mathrm{PPO}_{\text {mean }}$, and the overall changes in pulmonary $\dot{V} \mathrm{O}_{2}$ were analyzed using a paired-sample $t$ test for each intermittent performance 
test. Changes in plasma $\left[\mathrm{NO}_{2}^{-}\right]$recorded on days 3,4 , and 5 of supplementation were determined via a two-way (supplement $\times$ test) repeated-measures ANOVA. Likewise, alterations in power output, blood [lactate], and pulmonary $\dot{V} \mathrm{O}_{2}$ for work and recovery intervals in the $24 \times 6$-s, $7 \times 30$-s and $6 \times 60$-s protocols were assessed via two-way (supplement $\times$ interval) repeated-measures ANOVAs. Significant effects were further explored using Fisher's LSD. Statistical significance was accepted at $P<0.05$. Results are presented as mean \pm SD unless otherwise stated.

\section{Results}

Subjects reported that they consumed all servings of each supplement at the required times and that their diet and exercise habits prior to each experimental test were consistent.

\section{Plasma $\left[\mathrm{NO}_{2}^{-}\right]$}

The group mean plasma $\left[\mathrm{NO}_{2}{ }^{-}\right]$values obtained on days 3,4 , and 5 of the BR and PL supplementation periods are shown in Fig. 1. Plasma $\left[\mathrm{NO}_{2}^{-}\right]$was elevated during the BR supplementation compared to $\mathrm{PL}$ at all sample points $\left(P<0.05\right.$; Fig. 1a). The mean increase in plasma $\left[\mathrm{NO}_{2}{ }^{-}\right]$ with $\mathrm{BR}$ ingestion across the three sample points was $237 \%$ (BR $358 \pm 119$ vs. PL $106 \pm 32 \mathrm{nM} ; P<0.05$; Fig. 1b). Plasma $\left[\mathrm{NO}_{2}^{-}\right]$was not significantly different across days $3-5$ in PL or BR ( $P>0.05$; Fig. 1a).

\section{Power output}

The power output data during the $24 \times 6$-s, $7 \times 30$-s and $6 \times 60$-s protocols are illustrated in Figs. 2, 3, and 4, respectively.

\section{$24 \times 6$-s protocol}

There was no significant difference in $\mathrm{PPO}_{\text {mean }}$ between $\mathrm{BR}(792 \pm 159 \mathrm{~W})$ and PL $(782 \pm 154 \mathrm{~W} ; P>0.05)$. However, compared to PL, $\mathrm{MPO}_{\text {mean }}$ was significantly greater following BR supplementation (BR $568 \pm 136$ vs. PL $539 \pm 136 \mathrm{~W} ; P<0.05$; Fig. 2a). Further analyses revealed that BR supplementation did not significantly increase MPO in any individual sprints $(P>0.05)$. However, when the $24 \times 6$-s sprints were pooled into four groups of six sprints, MPO was greater with BR in sprints $1-6$ (BR $694 \pm 125$ vs. PL $647 \pm 122 \mathrm{~W} ; P<0.05$ ), but not in sprints $7-12$ (BR $560 \pm 100$ vs. PL $539 \pm 112 \mathrm{~W}$; $P>0.05$ ), $13-18$ (BR $518 \pm 111$ vs. PL $492 \pm 121 \mathrm{~W}$; $P>0.05$ ), and 19-24 (BR $500 \pm 114$ vs. PL $477 \pm 119 \mathrm{~W}$; $P>0.05$; Fig. 2b).
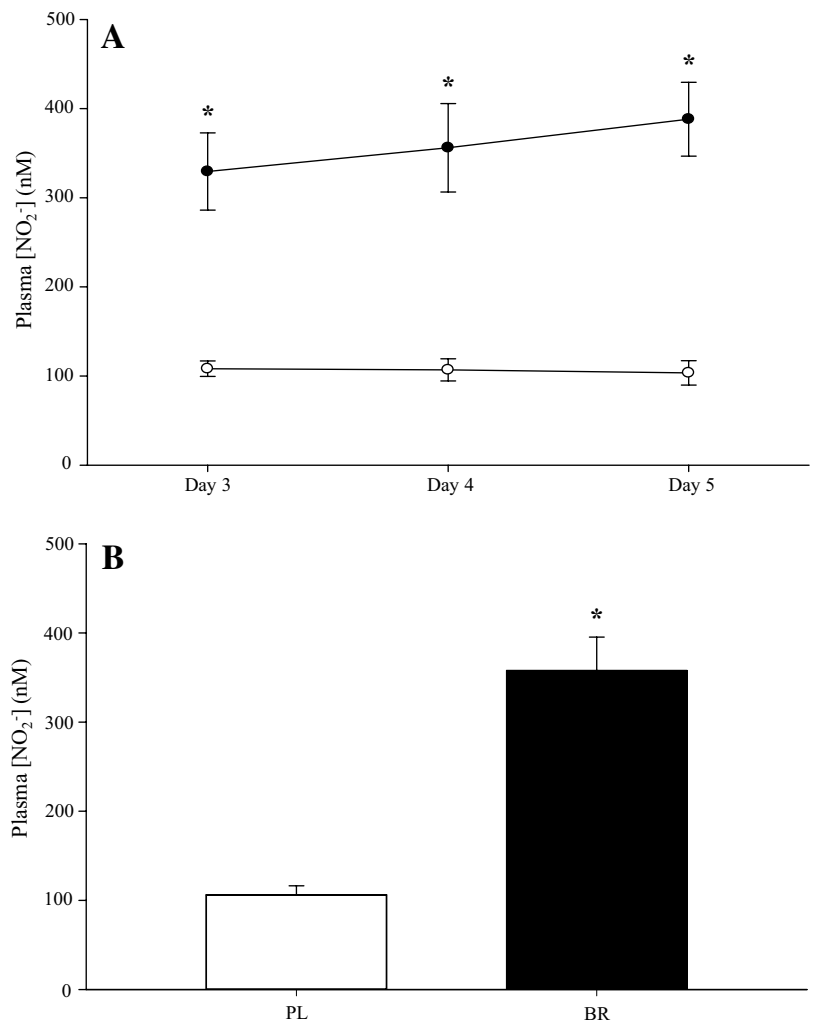

Fig. 1 Plasma nitrite concentration $\left(\left[\mathrm{NO}_{2}{ }^{-}\right]\right)$was elevated in $\mathrm{BR}$ (closed circles) compared to PL (open circles) on days 3, 4 and 5 of supplementation (a). On average across the three sample points, plasma $\left[\mathrm{NO}_{2}{ }^{-}\right.$] was $237 \%$ higher in BR (filled bar) compared to PL (open bar) (b). Error bar indicates the SE. $* P<0.05$ compared to PL

$7 \times 30$-s protocol

There were no significant differences between $B R$ and $\mathrm{PL}$ in $\mathrm{PPO}_{\text {mean }}$ (BR $768 \pm 157$ vs. PL $776 \pm 142 \mathrm{~W}$; $P>0.05$ ) or $\mathrm{MPO}_{\text {mean }}$ (BR $558 \pm 95$ vs. PL $562 \pm 94 \mathrm{~W}$; $P>0.05$; Fig. 3a) during the $7 \times 30$-s sprint exercise tests. There were also no differences between PL and $\mathrm{BR}$ in MP (Fig. 3b) and PP across individual sprints $(P>0.05)$.

$6 \times 60$-s protocol

The $\mathrm{MPO}_{\text {mean }}$ was not significantly different between PL $(375 \pm 59 \mathrm{~W})$ and BR $(374 \pm 57 \mathrm{~W} ; P>0.05$; Fig. $4 a)$ in the $6 \times 60$-s exercise test. There were no differences in MP between PL and BR for any of the individual intervals $(P>0.05$; Fig. 4b).

\section{Pulmonary gas exchange}

Pulmonary gas exchange data collected during the three intermittent performance tests in $\mathrm{BR}$ and $\mathrm{PL}$ are 

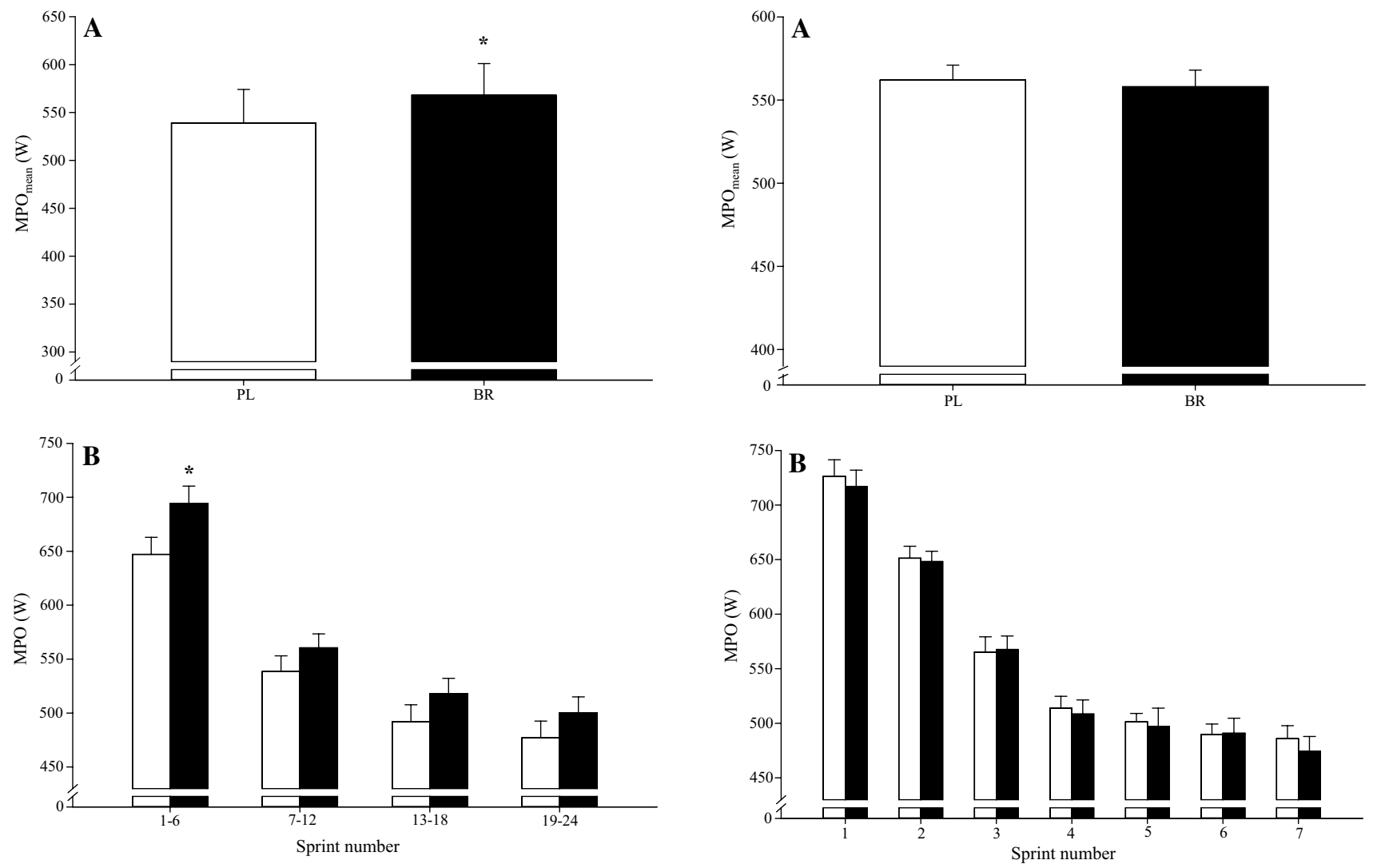

Fig. 2 The mean power output $\left(\mathrm{MPO}_{\text {mean }}\right)$ across the $24 \times 6$-s sprint protocol was $5 \%$ greater with BR (filled bars) relative to PL (open bars) (a). Specifically, mean power output (MPO) was greater with BR in sprints $1-6$, but not in sprints $7-12,13-18$ or $19-24$, when compared to PL (b). Error bars indicate the SE. $* P<0.05$ compared to PL

displayed in Table 1 . While pulmonary $\dot{V} \mathrm{O}_{2}$ and $\dot{V} \mathrm{CO}_{2}$ increased with time across the three intermittent exercise protocols $(P<0.05)$, mean pulmonary $\dot{V} \mathrm{O}_{2}$ and $\dot{V} \mathrm{CO}_{2}$ was not different between PL and BR during the work intervals, recovery periods or across the overall protocol in any of the exercise test protocols (all $P>0.05$; Table 1). RER was not different between PL and BR during the work intervals, recovery periods or across the overall protocol in the $7 \times 30$-s and $6 \times 60$-s protocols (all $P>0.05$; Table 1). However, compared to PL, RER was increased during the work intervals, recovery periods, and across the overall protocol in the $24 \times 6$-s protocol (all $P<0.05$; Table 1).

\section{Blood [lactate]}

Blood [lactate] was not significantly different between BR and PL at baseline (before completion of the warm-up procedure) in any test protocol (all $P>0.05$ ). The change in blood [lactate] during the intermittent exercise tests with BR and PL is shown in Fig. 5.

Fig. 3 The mean power output $\left(\mathrm{MPO}_{\text {mean }}\right)$ across the $7 \times 30$-s protocol (a) and mean power output (MPO) during each individual sprint (b) were not different between BR (filled bars) and PL (open bars). Error bars indicate the SE

\section{$24 \times 6$-s protocol}

The change in blood [lactate] from pre-exercise to the completion of sprint 24 was significantly greater in BR compared to PL (BR $7.3 \pm 2.2$ vs. PL $6.2 \pm 2.1 \mathrm{mM} ; P<0.05$; Fig. 5a). The rise in blood [lactate] above the pre-exercise value was also significantly greater with $\mathrm{BR}$ after sprint 22 $(P<0.05)$, but not in the earlier sprints $(P>0.05$; Fig. 5a).

\section{$7 \times 30-$ s protocol}

The change in blood [lactate], from pre-exercise to the completion of sprint 7 , was significantly greater in BR compared to PL (BR $10.5 \pm 1.8$ vs. PL $9.5 \pm 1.9 \mathrm{mM}$; $P<0.05$; Fig. 5b). BR supplementation also resulted in a significantly greater $\Delta$ blood [lactate] post-sprint 1 (BR $3.8 \pm 1.3$ vs. PL $2.0 \pm 0.9 \mathrm{mM} ; P<0.05)$, but not postsprints 2-6 ( $P>0.05$; Fig. $5 b)$.

\section{$6 \times 60-s$ protocol}

The change in blood [lactate], from baseline, to post completion of interval 6 , was not significantly different 

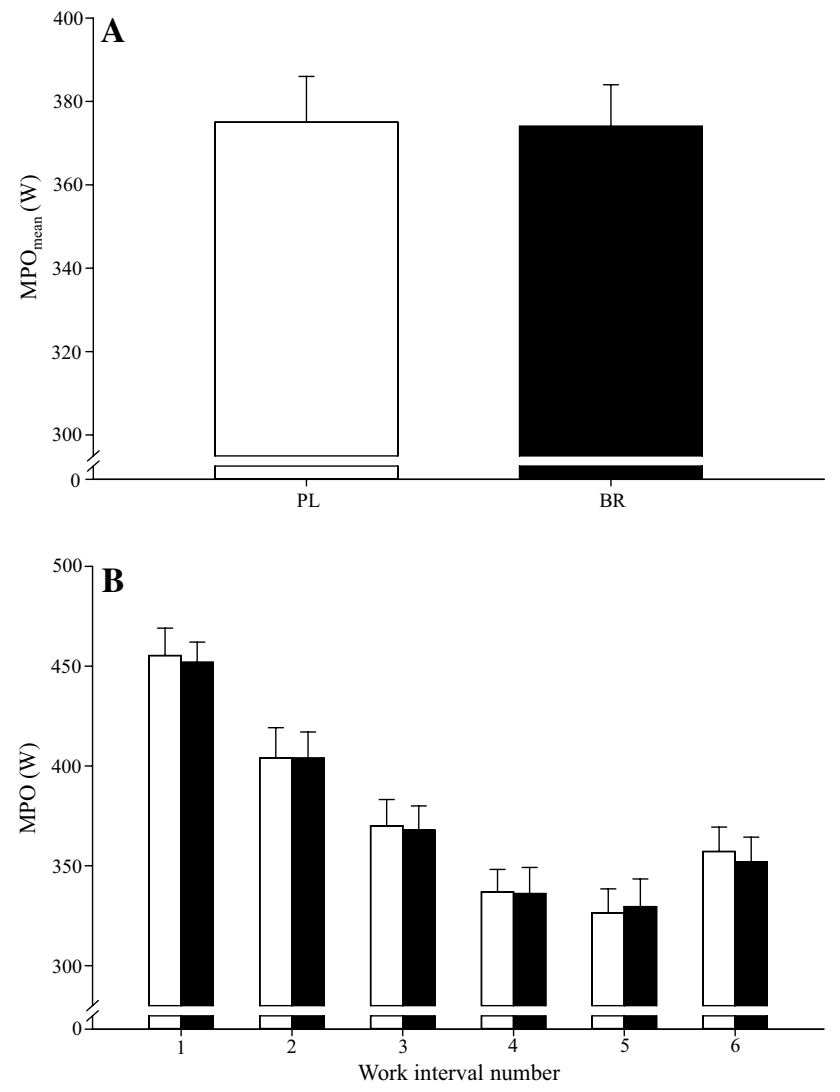

Fig. 4 The mean power output $\left(\mathrm{MPO}_{\text {mean }}\right)$ across the $6 \times 60$-s protocol (a) and mean power output (MPO) during each individual exercise interval (b) were not different between BR (filled bars) and PL (open bars). Error bars indicate the SE between supplements (BR $9.5 \pm 1.8$ vs. PL $8.6 \pm 1.6 \mathrm{mM}$; $P=0.07$; Fig. 5c). Furthermore, $\Delta$ blood [lactate] was not significantly impacted by BR post-intervals $1-5(P>0.05$; Fig. 5c).

\section{Discussion}

The principal original finding of this study was that shortterm supplementation with BR significantly improved mean power output during 24 6-s all-out sprints interspersed with $24 \mathrm{~s}$ of recovery but not during protocols comprising seven 30 -s all-out sprint efforts interspersed with 4 min of recovery or six 60-s self-paced maximal efforts interspersed with $60 \mathrm{~s}$ of recovery. BR was especially effective in improving $\mathrm{MPO}_{\text {mean }}$ in the early part of the $24 \times 6$-s protocol, with the difference between conditions being significantly different in the first 6 but not the subsequent 18 sprints. These findings suggest that BR supplementation may improve performance during high-intensity intermittent exercise when short-duration, maximal-intensity intervals are repeated with a short recovery duration, but not when interval and recovery durations are longer.

Consistent with previous studies, the circulating plasma $\left[\mathrm{NO}_{2}{ }^{-}\right]$was significantly increased following the shortterm BR supplementation regimen employed in this study (Bailey et al. 2009, 2010, 2015; Breese et al. 2013; Thompson et al. 2015; Vanhatalo et al. 2011; Wylie et al. 2012, 2013a, b). Importantly, plasma $\left[\mathrm{NO}_{2}^{-}\right]$was elevated above the corresponding PL trials by a similar magnitude on each

Table 1 Mean $( \pm S D)$ pulmonary gas exchange variables during the work intervals, recovery periods, and across the overall protocol in all three exercise test protocols following PL and BR supplementation

\begin{tabular}{|c|c|c|c|c|c|c|}
\hline & \multicolumn{2}{|c|}{$24 \times 6$-s protocol } & \multicolumn{2}{|c|}{$7 \times 30$-s protocol } & \multicolumn{2}{|c|}{$6 \times 60$-s protocol } \\
\hline & PL & BR & PL & $\mathrm{BR}$ & PL & $\mathrm{BR}$ \\
\hline \multicolumn{7}{|l|}{$\dot{V} \mathrm{O}_{2}$} \\
\hline Work interval $\left(\mathrm{L} \mathrm{min}^{-1}\right)$ & $3.66 \pm 0.29$ & $3.64 \pm 0.31$ & $2.57 \pm 0.22$ & $2.55 \pm 0.26$ & $3.33 \pm 0.23$ & $3.27 \pm 0.32$ \\
\hline Recovery period $\left(\mathrm{L} \mathrm{min}^{-1}\right)$ & $3.11 \pm 0.24$ & $3.12 \pm 0.28$ & $1.64 \pm 0.15$ & $1.66 \pm 0.16$ & $2.73 \pm 0.17$ & $2.70 \pm 0.28$ \\
\hline Overall $\left(\mathrm{L} \mathrm{min}^{-1}\right)$ & $3.22 \pm 0.24$ & $3.23 \pm 0.28$ & $1.74 \pm 0.15$ & $1.76 \pm 0.16$ & $3.02 \pm 0.18$ & $2.99 \pm 0.29$ \\
\hline \multicolumn{7}{|l|}{$\dot{V} \mathrm{CO}_{2}$} \\
\hline Work interval $\left(\mathrm{L} \mathrm{min}^{-1}\right)$ & $4.11 \pm 0.32$ & $4.27 \pm 0.41$ & $2.50 \pm 0.23$ & $2.49 \pm 0.30$ & $3.61 \pm 0.31$ & $3.57 \pm 0.36$ \\
\hline Recovery period $\left(\mathrm{L} \min ^{-1}\right)$ & $3.39 \pm 0.28$ & $3.52 \pm 0.32$ & $2.00 \pm 0.16$ & $2.02 \pm 0.18$ & $3.50 \pm 0.27$ & $3.50 \pm 0.28$ \\
\hline Overall $\left(\mathrm{L} \min ^{-1}\right)$ & $3.53 \pm 0.28$ & $3.67 \pm 0.33$ & $2.05 \pm 0.16$ & $2.07 \pm 0.18$ & $3.55 \pm 0.27$ & $3.53 \pm 0.32$ \\
\hline \multicolumn{7}{|l|}{ RER } \\
\hline Work interval & $1.13 \pm 0.01$ & $1.18 \pm 0.04^{*}$ & $1.04 \pm 0.04$ & $1.04 \pm 0.06$ & $1.12 \pm 0.03$ & $1.13 \pm 0.03$ \\
\hline Recovery period & $1.09 \pm 0.02$ & $1.13 \pm 0.05^{*}$ & $1.26 \pm 0.06$ & $1.26 \pm 0.04$ & $1.33 \pm 0.10$ & $1.35 \pm 0.10$ \\
\hline Overall & $1.10 \pm 0.02$ & $1.14 \pm 0.05^{*}$ & $1.24 \pm 0.05$ & $1.23 \pm 0.04$ & $1.23 \pm 0.06$ & $1.24 \pm 0.06$ \\
\hline
\end{tabular}

* Significantly different from PL $(P<0.05)$ 

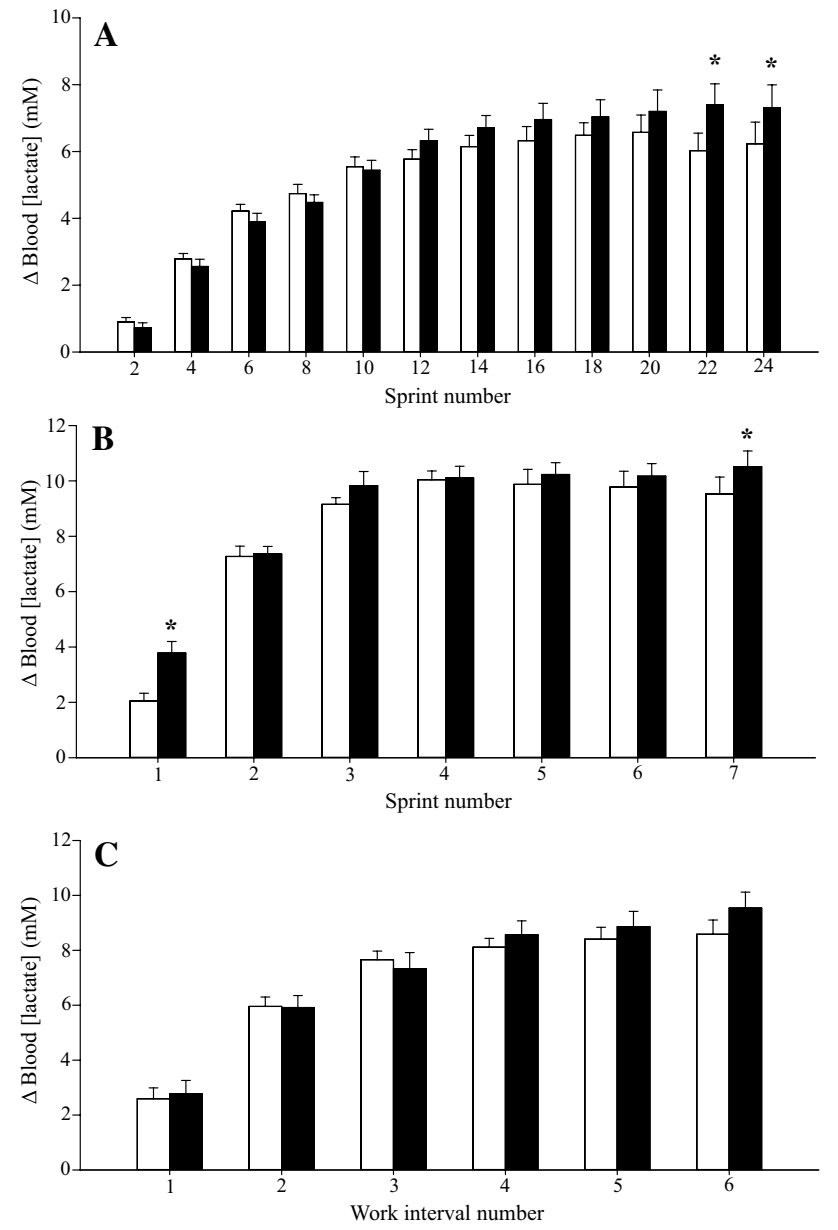

Fig. 5 Change $(\Delta)$ relative to pre-exercise baseline in blood lactate concentration ([lactate]) during the $24 \times 6$-s (a), $7 \times 30$-s (b) and $6 \times 60$-s (c) exercise protocols, following PL (open bars) and BR (filled bars) supplementation. Note the greater $\boldsymbol{\Delta}$ in blood [lactate] with BR after sprints 22 and 24 in the $24 \times 6$-s protocol, and after sprints 1 and 7 in the $7 \times 30$-s protocol. Error bars indicate the SE. $* P<0.05$ compared to PL

of the BR trials. Therefore, the relative efficacy of BR to improve performance in the intermittent tests cannot be attributed to differences in plasma $\left[\mathrm{NO}_{2}{ }^{-}\right]$between tests. An increase in plasma $\left[\mathrm{NO}_{2}{ }^{-}\right]$represents an increased 'substrate' for $\mathrm{NO}$ synthesis through the $\mathrm{O}_{2}$-independent reduction of $\mathrm{NO}_{2}{ }^{-}$to $\mathrm{NO}$ (Lundberg and Weitzberg 2009). A comparable BR-induced increase in plasma $\left[\mathrm{NO}_{2}{ }^{-}\right]$has previously been reported to enhance performance in continuous sub-maximal endurance exercise, at least in recreationally active/moderately trained subjects (e.g., Bailey et al. 2015; Breese et al. 2013; Vanhatalo et al. 2011; Wylie et al. 2013a). However, it is important to note that the reduction of $\mathrm{NO}_{2}{ }^{-}$to $\mathrm{NO}$ is enhanced as $\mathrm{PO}_{2}$ (Castello et al. 2006) and pH (Modin et al. 2001) decline, and recent reports suggest that the physiological and performance effects of $\mathrm{NO}_{3}{ }^{-}$supplementation are enhanced in type II muscle compared to type I muscle or when type II fiber recruitment is expected to be greater (Bailey et al. 2015; Breese et al. 2013; Coggan et al. 2015; Ferguson et al. 2013, 2015; Hernández et al. 2012). Therefore, given that muscle $\mathrm{PO}_{2}$ and $\mathrm{pH}$ decline (Richardson et al. 1995) and the recruitment of type II muscle is greater (Essén 1978; Green 1978; Krustrup et al. 2004, 2009; Thomson et al. 1979) as exercise intensity increases, we reasoned that BR supplementation would be effective in enhancing performance during the intermittent high-intensity exercise protocols employed in this study.

Consistent with our experimental hypothesis and some previous reports of improved intermittent exercise performance after BR supplementation (Aucouturier et al. 2015; Bond et al. 2012; Thompson et al. 2015; Wylie et al. 2013a), performance (MPO) in the $24 \times 6$-s protocol was $5 \%$ greater with BR relative to PL. When the $24 \times 6-\mathrm{s}$ protocol was divided into $25 \%$ completion segments, MPO was significantly improved with BR in sprints 1-6 $(+7 \%)$, but not in sprints $7-12(+4 \% ; P=0.18), 13-18$ $(+5 \% ; P=0.12)$ or $19-24(+5 \% ; P=0.14)$. These findings are consistent with recent observations that BR supplementation increased total work done during the first of two 40-min halves comprising repeated 2-min blocks of a 6-s all-out sprint, 100-s active recovery, and $20 \mathrm{~s}$ of rest (Thompson et al. 2015). However, in contrast to our experimental hypothesis, performance was not significantly impacted by BR supplementation in the $7 \times 30$-s or $6 \times 60$-s intermittent exercise protocols. Some previous studies have also reported no improvement in high-intensity intermittent exercise performance following BR supplementation (Christensen et al. 2013; Martin et al. 2014; Muggeridge et al. 2013). It should be acknowledged that, since subjects were asked to complete the three intermittent exercise protocols on consecutive days within each supplementation period, it is possible that basal fatigue increased across the series of tests and that this impacted performance during the second and/or third test days. However, since the participants were accustomed to high-intensity intermittent exercise, it is unlikely that changes in basal fatigue resistance would have substantially impacted our results.

Inconsistency in the efficacy of BR supplementation to improve high-intensity intermittent exercise performance in previous studies can be attributed, in part, to inter-study differences in participant training status, exercise modality, exercise protocol, and $\mathrm{NO}_{3}{ }^{-}$supplementation procedures. Indeed, studies that have reported no improvement in intermittent exercise performance after BR supplementation have tested highly trained endurance athletes (Christensen et al. 2013; Muggeridge et al. 2013) and/or administered a low $\left(<5 \mathrm{mmol} \mathrm{NO}_{3}{ }^{-}\right)$acute dose of BR (Martin et al. 2014; Muggeridge et al. 2013). In contrast, studies that have reported improved intermittent exercise performance 
following BR supplementation have involved recreationally active or moderately trained participants (Aucouturier et al. 2015; Thompson et al. 2015; Wylie et al. 2013b) and/or administered more chronic ( $\geq 3$ days) BR supplementation (Aucouturier et al. 2015; Bond et al. 2012; Thompson et al. 2015) or a large $\left(\sim 29 \mathrm{mmol} \mathrm{NO}_{3}^{-}\right)$dose of BR administered over $24 \mathrm{~h}$ (Wylie et al. 2013b). Another complication when interpreting the existing literature with regard to the efficacy of BR supplementation is that previous studies employed single intermittent exercise performance tests that have differed considerably with regard to work and rest intensities, work and rest durations, work-to-rest ratio, and number of work intervals (Aucouturier et al. 2015; Bond et al. 2012; Christensen et al. 2013; Martin et al. 2014; Muggeridge et al. 2013; Thompson et al. 2015; Wylie et al. 2013a). The findings of the present study therefore make an important contribution to our understanding of the effectiveness of BR supplementation to improve performance in different intermittent exercise protocols.

In contrast to the reduced steady-state $\dot{V} \mathrm{O}_{2}$ that has previously been observed during submaximal constant work rate exercise following nitrate supplementation (e.g. Bailey et al. 2009; Larsen et al. 2007; Wylie et al. 2013a; see Jones 2014 for review), $\dot{V} \mathrm{O}_{2}$ was not altered during any of the intermittent exercise protocols in the present study, which is consistent with our recent observations (Thompson et al. 2015). Therefore, the improved intermittent exercise performance in the $24 \times 6$-s protocol was not a function of changes in whole-body $\mathrm{O}_{2}$ consumption. Given reports that $\mathrm{NO}_{3}{ }^{-}$supplementation is more effective at enhancing physiological responses and performance in type II compared to type I muscle (Ferguson et al. 2013, 2015; Hernández et al. 2012) or in situations where type II muscle fiber recruitment is expected to be greater (Bailey et al. 2015; Breese et al. 2013; Coggan et al. 2015), the differing effects of BR supplementation on performance in the different intermittent exercise protocols in the present study might be linked to differences in the muscle fiber recruitment patterns, and contribution to force production, across the different protocols tested. Studies assessing muscle fiber recruitment patterns from single-fiber high-energy phosphate depletion suggest that the recruitment of type II muscle is greater in $6 \mathrm{~s}$ sprints than $30 \mathrm{~s}$ sprints (Casey et al. 1996; Esbjörnsson-Liljedahl et al. 1999; Gray et al. 2008; Karatzaferi et al. 2001). The improved performance in the $24 \times 6$-s protocol, but not the $7 \times 30$-s or $6 \times 60$-s protocols, might therefore be linked to improved force production of type II muscle (Coggan et al. 2015; Hernández et al. 2012) as a consequence of increased sarcoplasmic reticulum calcium release (Hernández et al. 2012) and/or improved perfusion/ oxygenation (Ferguson et al. 2013, 2015) in type II muscle. Increased perfusion of type II muscle would be particularly important given that the decline in muscle $\mathrm{PCr}$ is an important determinant of fatigue development during maximal-intensity intermittent exercise (Fulford et al. 2013; Gaitanos et al. 1993) and that muscle $\mathrm{PCr}$ resynthesis in recovery is an $\mathrm{O}_{2}$-dependent process (Trump et al. 1996; Vanhatalo et al. 2011). However, given that type II fibers are also heavily recruited during a 30-s all-out cycling sprint (Esbjörnsson-Liljedahl et al. 1999), it is unclear why dietary nitrate supplementation did not improve performance, at least during the first 30-s sprint, in the $7 \times 30$-s protocol.

A novel observation was that the increase in blood [lactate] from baseline to the end of exercise was greater after BR supplementation in the $24 \times 6$-s and $7 \times 30$-s protocols, although this was not the case in the $6 \times 60$-s protocol. Interestingly, RER was also significantly elevated after BR supplementation in the $24 \times 6$-s protocol. It is uncertain to what extent these changes contributed to enhanced performance with BR. In the $7 \times 30$-s protocol, performance was not enhanced despite the greater blood [lactate]; in the $24 \times 6$-s protocol, blood [lactate] was only greater with BR after sprints 22 and 24 where performance was not significantly enhanced (although it should be noted that there will be a temporal lag between muscle lactate production and the appearance of lactate in the blood; Jorfeldt et al. 1978). It has previously been reported that BR supplementation does not influence muscle $\mathrm{pH}$ during maximal-intensity intermittent exercise (Fulford et al. 2013) or alter glycolytic ATP turnover during continuous sub-maximal exercise (Bailey et al. 2010). Therefore, the increased blood lactate accumulation during the $24 \times 6$-s and $7 \times 30$-s protocols after BR supplementation may not necessarily reflect increased ATP flux through anaerobic glycolysis, but additional research is required before this possibility can be excluded. Instead, the increased blood lactate accumulation during these tests might be a function of increased perfusion of type II muscle following BR supplementation (Ferguson et al. 2013, 2015) since lactate production is greater in type II muscle (EsbjörnssonLiljedahl et al. 1999) and lactate efflux can be increased with a greater muscle perfusion (Juel 1997). Further research is required to investigate the underlying mechanisms for the improved performance during short-duration maximal-intensity intermittent exercise following BR supplementation.

The present study provides important new insights into the efficacy of BR supplementation to improve performance in different types of intermittent exercise, and specifically indicates that BR supplementation enhanced performance during the $24 \times 6$-s protocol in which 6-s sprints were separated by $24 \mathrm{~s}$ of recovery. Repeated bouts of short-duration, high-intensity exercise interspersed with brief recovery intervals is a hallmark of many invasion games such as Association football, rugby union/league, and field hockey (King et al. 2009; Mohr et al. 2003; Spencer et al. 2004). It is interesting, 
therefore, that BR supplementation was most effective at enhancing performance in the intermittent exercise test that most closely resembled the exercise patterns manifest during many team sports, findings which are consistent with Thompson et al. (2015). Although performance was only significantly improved (by $7 \%$ ) after BR supplementation over the first six sprints of the $24 \times 6$-s protocol, it is possible that the non-significant $4-5 \%$ increase in power output over the remaining 18 sprints might represent a practically meaningful improvement in performance in intermittent team sports. However, further research using validated field tests is required to assess the potential of dietary nitrate supplementation to improve team sport performance. Moreover, it should be acknowledged that a larger sample size than was used in the present study might be required to detect changes that are small but potentially practically meaningful in shortduration high-intensity intermittent exercise performance after BR supplementation.

In conclusion, the results of this study suggest that short-term BR supplementation, which increased the circulating plasma $\left[\mathrm{NO}_{2}{ }^{-}\right]$, can improve performance during 24 6-s all-out sprints interspersed with $24 \mathrm{~s}$ of recovery. This improvement arose chiefly as a result of a specific performance enhancement over the first six sprints of this test protocol. However, despite a comparable increase in plasma $\left[\mathrm{NO}_{2}^{-}\right]$, BR supplementation did not significantly improve performance during seven 30 -s all-out sprints interspersed with 4 min of recovery or six 60 -s self-paced maximal efforts interspersed with $60 \mathrm{~s}$ of recovery. These findings elucidate the conditions under which BR supplementation may be ergogenic during high-intensity intermittent exercise and invite further mechanistic and practical exposition.

Open Access This article is distributed under the terms of the Creative Commons Attribution 4.0 International License (http://creativecommons.org/licenses/by/4.0/), which permits unrestricted use, distribution, and reproduction in any medium, provided you give appropriate credit to the original author(s) and the source, provide a link to the Creative Commons license, and indicate if changes were made.

\section{References}

Aucouturier J, Boissière J, Pawlak-Chaouch M, Cuvelier G, Gamelin FX (2015) Effect of dietary nitrate supplementation on tolerance to supramaximal intensity intermittent exercise. Nitric Oxide 49:16-25. doi:10.1016/j.niox.2015.05.004

Bailey SJ, Winyard P, Vanhatalo A, Blackwell JR, Dimenna FJ, Wilkerson DP, Tarr J, Benjamin N, Jones AM (2009) Dietary nitrate supplementation reduces the $\mathrm{O}_{2}$ cost of low-intensity exercise and enhances tolerance to high-intensity exercise in humans. J Appl Physiol 107:1144-1155. doi:10.1152/ japplphysiol.00722.2009
Bailey SJ, Fulford J, Vanhatalo A, Winyard PG, Blackwell JR, DiMenna FJ, Wilkerson DP, Benjamin N, Jones AM (2010) Dietary nitrate supplementation enhances muscle contractile efficiency during knee-extensor exercise in humans. J Appl Physiol 109:135-148. doi:10.1152/japplphysiol.00046.2010

Bailey SJ, Varnham RL, DiMenna FJ, Breese BC, Wylie LJ, Jones AM (2015) Inorganic nitrate supplementation improves muscle oxygenation, $\mathrm{O}_{2}$ uptake kinetics, and exercise tolerance at high but not low pedal rates. J Appl Physiol 118:1396-1405. doi:10.1152/japplphysiol.01141.2014

Bogdanis GC, Nevill ME, Boobis LH, Lakomy HK, Nevill AM (1995) Recovery of power output and muscle metabolites following $30 \mathrm{~s}$ of maximal sprint cycling in man. J Physiol 482:467-480

Bogdanis GC, Nevill ME, Boobis LH, Lakomy HK (1996) Contribution of phosphocreatine and aerobic metabolism to energy supply during repeated sprint exercise. J Appl Physiol 80:876-884

Bond H, Morton L, Braakhuis AJ (2012) Dietary nitrate supplementation improves rowing performance in well-trained rowers. Int $\mathbf{J}$ Sport Nutr Exerc Metab 22:251-256

Breese BC, McNarry MA, Marwood S, Blackwell JR, Bailey SJ, Jones AM (2013) Beetroot juice supplementation speeds $\mathrm{O}_{2}$ uptake kinetics and improves exercise tolerance during severeintensity exercise initiated from an elevated metabolic rate. Am J Physiol Regul Integr Comp Physiol 305:1441-1550. doi:10.1152/ajpregu.00295.2013

Casey A, Constantin-Teodosiu D, Howell S, Hultman E, Greenhaff PL (1996) Metabolic response of type I and II muscle fibers during repeated bouts of maximal exercise in humans. Am J Physiol 271:38-43

Castello PR, David PS, McClure T, Crook Z, Poyton RO (2006) Mitochondrial cytochrome oxidase produces nitric oxide under hypoxic conditions: implications for oxygen sensing and hypoxic signaling in eukaryotes. Cell Metab 3:277-287

Cermak NM, Gibala MJ, van Loon LJ (2012) Nitrate supplementation's improvement of 10-km time-trial performance in trained cyclists. Int J Sport Nutr Exerc Metab 22:64-71

Chidnok W, DiMenna FJ, Fulford J, Bailey SJ, Skiba PF, Vanhatalo A, Jones AM (2013) Muscle metabolic responses during highintensity intermittent exercise measured by ${ }^{31} \mathrm{P}-\mathrm{MRS}$ : relationship to the critical power concept. Am J Physiol Regul Integr Comp Physiol 305:1085-1092. doi:10.1152/ajpregu.00406.2013

Christensen PM, Nyberg M, Bangsbo J (2013) Influence of nitrate supplementation on $\mathrm{VO}_{2}$ kinetics and endurance of elite cyclists. Scand J Med Sci Sports 23:21-31. doi:10.1111/sms.12005

Coggan AR, Leibowitz JL, Kadkhodayan A, Thomas DP, Ramamurthy S, Spearie CA, Waller S, Farmer M, Peterson LR (2015) Effect of acute dietary nitrate intake on maximal knee extensor speed and power in healthy men and women. Nitric Oxide 48:16-21. doi:10.1016/j.niox.2014.08.014

Esbjörnsson-Liljedahl M, Sundberg CJ, Norman B, Jansson E (1999) Metabolic response in type I and type II muscle fibers during a 30-s cycle sprint in men and women. J Appl Physiol 87:1326-1332

Essén B (1978) Glycogen depletion of different fibre types in human skeletal muscle during intermittent and continuous exercise. Acta Physiol Scand 103:446-455

Ferguson SK, Hirai DM, Copp SW, Holdsworth CT, Allen JD, Jones AM, Musch TI, Poole DC (2013) Impact of dietary nitrate supplementation via beetroot juice on exercising muscle vascular control in rats. J Physiol 591:547-557. doi:10.1113/ jphysiol.2012.243121

Ferguson SK, Holdsworth CT, Wright JL, Fees AJ, Allen JD, Jones AM, Musch TI, Poole DC (2015) Microvascular oxygen pressures in muscles comprised of different fiber types: impact of dietary nitrate supplementation. Nitric Oxide 48:38-43. doi:10.1016/j.niox.2014.09.157 
Fitzsimons M, Dawson B, Ware D, Wilkinson A (1993) Cycling and running tests of repeated sprint ability. Aust J Sci Med Sport 25:82-87

Fulford J, Winyard PG, Vanhatalo A, Bailey SJ, Blackwell JR, Jones AM (2013) Influence of dietary nitrate supplementation on human skeletal muscle metabolism and force production during maximum voluntary contractions. Pflugers Arch 465:517-528. doi:10.1007/s00424-013-1220-5

Gaitanos GC, Williams C, Boobis LH, Brooks S (1993) Human muscle metabolism during intermittent maximal exercise. J Appl Physiol 75:712-719

Govoni M, Jansson EA, Weitzberg E, Lundberg JO (2008) The increase in plasma nitrite after a dietary nitrate load is markedly attenuated by an antibacterial mouthwash. Nitric Oxide 19:333337. doi:10.1016/j.niox.2008.08.003

Gray SR, Söderlund K, Ferguson RA (2008) ATP and phosphocreatine utilization in single human muscle fibres during the development of maximal power output at elevated muscle temperatures. J Sports Sci 26:701-707. doi:10.1080/02640410701744438

Green HJ (1978) Glycogen depletion patterns during continuous and intermittent ice skating. Med Sci Sports 10:183-187

Haider G, Folland JP (2014) Nitrate supplementation enhances the contractile properties of human skeletal muscle. Med Sci Sports Exerc 46:2234-2243. doi:10.1249/MSS.0000000000000351

Hernández A, Schiffer TA, Ivarsson N, Cheng AJ, Bruton JD, Lundberg JO, Weitzberg E, Westerblad H (2012) Dietary nitrate increases tetanic $\left[\mathrm{Ca}^{2+}\right] \mathrm{i}$ and contractile force in mouse fast-twitch muscle. J Physiol 590:3575-3583. doi:10.1113/ jphysiol.2012.232777

Jones AM (2014) Dietary nitrate supplementation and exercise performance. Sports Med 44:S35-S45. doi:10.1007/ s40279-014-0149-y

Jorfeldt L, Juhlin-Dannfelt A, Karlsson J (1978) Lactate release in relation to tissue lactate in human skeletal muscle during exercise. J Appl Physiol Respir Environ Exerc Physiol 44:350-352

Juel C (1997) Lactate-proton cotransport in skeletal muscle. Physiol Rev 77:321-358

Karatzaferi C, de Haan A, Ferguson RA, van Mechelen W, Sargeant AJ (2001) Phosphocreatine and ATP content in human single muscle fibres before and after maximum dynamic exercise. Pflugers Arch 442:467-474

Kelly J, Vanhatalo A, Bailey SJ, Wylie LJ, Tucker C, List S, Winyard PG, Jones AM (2014) Dietary nitrate supplementation: effects on plasma nitrite and pulmonary $\mathrm{O}_{2}$ uptake dynamics during exercise in hypoxia and normoxia. Am J Physiol Regul Integr Comp Physiol 307:R920-R930. doi:10.1152/ajpregu.00068.2014

King T, Jenkins D, Gabbett T (2009) A time-motion analysis of professional rugby league match-play. J Sports Sci 27:213-219. doi:10.1080/02640410802538168

Krustrup P, Söderlund K, Mohr M, Bangsbo J (2004) The slow component of oxygen uptake during intense, sub-maximal exercise in man is associated with additional fibre recruitment. Pflugers Arch 447:855-866

Krustrup P, Söderlund K, Relu MU, Ferguson RA, Bangsbo J (2009) Heterogeneous recruitment of quadriceps muscle portions and fibre types during moderate intensity knee-extensor exercise: effect of thigh occlusion. Scand J Med Sci Sports 19:576-584. doi:10.1111/j.1600-0838.2008.00801.x

Larsen FJ, Ekblom B, Sahlin K, Lundberg JO, Weitzberg E (2007) Effects of dietary nitrate on oxygen cost during exercise. Acta Physiol 191:59-66. doi:10.1111/j.1748-1716.2007.01713.x

Lundberg JO, Weitzberg E (2009) NO generation from inorganic nitrate and nitrite: role in physiology, nutrition and therapeutics. Arch Pharm Res 32:1119-1126. doi:10.1007/s12272-009-1803-z
Martin K, Smee D, Thompson KG, Rattray B (2014) No improvement of repeated-sprint performance with dietary nitrate. Int J Sports Physiol Perform 9:845-850. doi:10.1123/ijspp.2013-0384

McCartney N, Obminski G, Heigenhauser GJ (1985) Torque-velocity relationship in isokinetic cycling exercise. J Appl Physiol 58:1459-1462

Mendez-Villanueva A, Edge J, Suriano R, Hamer P, Bishop D (2012) The recovery of repeated-sprint exercise is associated with $\mathrm{PCr}$ resynthesis, while muscle $\mathrm{pH}$ and EMG amplitude remain depressed. PLoS One 7:e51977. doi:10.1371/journal. pone.0051977

Modin A, Björne H, Herulf M, Alving K, Weitzberg E, Lundberg JO (2001) Nitrite-derived nitric oxide: a possible mediator of 'acidic-metabolic' vasodilation. Acta Physiol Scand 171:9-16

Mohr M, Krustrup P, Bangsbo J (2003) Match performance of highstandard soccer players with special reference to development of fatigue. J Sports Sci 21:519-528

Muggeridge DJ, Howe CCF, Spendiff O, Pedlar C, James PE, Easton C (2013) The effects of a single dose of concentrated beetroot juice on performance in trained flatwater kayakers. Int J Sport Nutr Exerc Metab 23:498-506

Porcelli S, Ramaglia M, Bellistri G, Pavei G, Pugliese L, Montorsi M, Rasica L, Marzorati M (2015) Aerobic fitness affects the exercise performance responses to nitrate supplementation. Med Sci Sports Exerc 47:1643-1651. doi:10.1249/ MSS.0000000000000577

Richardson RS, Noyszewski EA, Kendrick KF, Leigh JS, Wagner PD (1995) Myoglobin $\mathrm{O}_{2}$ desaturation during exercise. Evidence of limited $\mathrm{O}_{2}$ transport. J Clin Invest 96:1916-1926

Spencer M, Lawrence S, Rechichi C, Bishop D, Dawson B, Goodman C (2004) Time-motion analysis of elite field hockey, with special reference to repeated-sprint activity. J Sports Sci 22:843-850

Stamler JS, Meissner G (2001) Physiology of nitric oxide in skeletal muscle. Physiol Rev 81:209-237

Thompson C, Wylie LJ, Fulford J, Kelly J, Black MI, McDonagh ST, Jeukendrup AE, Vanhatalo A, Jones AM (2015) Dietary nitrate improves sprint performance and cognitive function during prolonged intermittent exercise. Eur J Appl Physiol 115:1825-1834

Thomson JA, Green HJ, Houston ME (1979) Muscle glycogen depletion patterns in fast twitch fibre subgroups of man during submaximal and supramaximal exercise. Pflugers Arch 379:105-108

Trump ME, Heigenhauser GJ, Putman CT, Spriet LL (1996) Importance of muscle phosphocreatine during intermittent maximal cycling. J Appl Physiol 80:1574-1580

Vanhatalo A, Fulford J, Bailey SJ, Blackwell JR, Winyard PG, Jones AM (2011) Dietary nitrate reduces muscle metabolic perturbation and improves exercise tolerance in hypoxia. J Physiol 589:5517-5528. doi:10.1113/jphysiol.2011.216341

Waldron M, Highton J (2014) Fatigue and pacing in high-intensity intermittent team sport: an update. Sports Med 44:1645-1658. doi:10.1007/s40279-014-0230-6

Wylie LJ, Kelly J, Bailey SJ, Blackwell JR, Skiba PF, Winyard PG, Jeukendrup AE, Vanhatalo A, Jones AM (2013a) Beetroot juice and exercise: pharmacodynamic and dose-response relationships. J Appl Physiol 115:325-336. doi:10.1152/ japplphysiol.00372.2013

Wylie LJ, Mohr M, Krustrup P, Jackman SR, Ermıdis G, Kelly J, Black MI, Bailey SJ, Vanhatalo A, Jones AM (2013b) Dietary nitrate supplementation improves team sport-specific intense intermittent exercise performance. Eur J Appl Physiol 113:16731684. doi:10.1007/s00421-013-2589-8 